\title{
JóVENES, CONTEXTOS DE VIOLENCIA ESTRUCTURAL Y CIUDADANÍA
}

\author{
ALICIA TINOCO-GARCÍA ${ }^{1}$ \\ ABRAHAM OSORIO BALLESTEROS ${ }^{2}$ \\ FELIPE GONZÁLEZ ORTIZ ${ }^{3}$
}

\begin{abstract}
RESUMEN
El objetivo del artículo es reflexionar sobre las condiciones de vulnerabilidad y riesgo en las que viven miles de jóvenes en el Estado de México a causa de la violencia estructural que los aqueja y excluye, negándoles la ciudadanía. Para ello recuperamos, en términos panorámicos, indicadores socioeconómicos para los jóvenes en el mundo, como datos de violencia estructural; realizamos un acercamiento a las Preceptorías Juveniles Regionales de Reintegración Social del Estado de México a través de cuatro entrevistas semiestructuradas, y dos reportes de observaciones de campo en dos torneos, uno de fútbol y otro de ajedrez, en los que participaron jóvenes de las Preceptorías.
\end{abstract}

\footnotetext{
${ }^{1}$ Doctora en Ciencias Sociales. Profesora-Investigadora de la Facultad de Ciencias Políticas y Sociales, Universidad Autónoma del Estado de México. Correo electrónico: almatiga@gmail.com

${ }^{2}$ Doctor en Investigación en Ciencias Sociales con mención en Sociología por la FLACSO México. Profesor-Investigador de tiempo completo de la Facultad de Ciencias Políticas y Sociales, Universidad Autónoma del Estado de México. Correo electrónico: sub_abraham@yahoo.com.mx

${ }^{3}$ Doctor en Antropología, Profesor-Investigador de la Facultad de Ciencias Políticas y Sociales, Universidad Autónoma del Estado de México. Correo electrónico: felsus1@yahoo.es
} 
Asimismo, planteamos que las Preceptorías, al final, son un eslabón más de la violencia estructural e institucional.

PALABRAS CLAVE: JÓVENES, VIOLENCIA ESTRUCTURAL, CIUDADANÍA

\title{
JOVENS, CONTEXTOS DE VIOLÊNCIA ESTRUTURAL E CIDADANIA
}

\begin{abstract}
RESUMO
O objetivo do artigo é refletir sobre as condições de vulnerabilidade e risco em que vivem milhares de jovens no Estado do México, por causa da violência estrutural que os afeta e os exclui negando sua cidadania. Para isso recuperamos, em termos panorâmicos, indicadores socioeconômicos para os jovens no mundo, como dados sobre a violência estrutural; realizamos uma aproximação às Preceptorias Juvenis Regionais de Reintegração Social do Estado do México através de quatro entrevistas semiestruturadas, e dois relatórios de observação de campo em dois torneios, um de futebol e o outro de xadrez nos que participaram jovens das Preceptorias. Também consideramos que as Preceptorias, no final, são um elo mais da violência estrutural e institucional.

PALAVRAS-CHAVE: JOVENS, VIOLÊNCIA ESTRUTURAL, CIDADANIA

\section{YOUNG PEOPLE, STRUCTURAL VIOLENCE CONTEXTS AND CITIZENSHIP}

\begin{abstract}
This article is aimed at reflecting on the conditions of vulnerability and risk in which thousands of young people live in the State of Mexico as a result of the structural violence that afflicts and excludes them by denying their citizenship. For this, we recovered, in panoramic terms, socio-economic indicators for young people in the world
\end{abstract}


such as data on structural violence; we made an approach to the Preceptories of Regional Juvenile Reintegration from the State of Mexico through four semi-structured interviews and two field observation reports on two tournaments (football and chess), in which young people from the Preceptories participated. We state, as a conclusion, that Preceptories are, eventually, no more than another link of structural and institutional violence.

KEYWORDS: YOUNG PEOPLE, STRUCTURAL VIOLENCE, CITIZENSHIP

\section{INTRODUCCIÓN}

El objetivo del artículo es reflexionar sobre las condiciones de vulnerabilidad y riesgo en que viven miles de jóvenes en el Estado de México a causa de la violencia estructural que los aqueja y excluye, negándoles una mínima ciudadanía, es decir, contar con los mínimos civiles que permitirían el despliegue de sus capacidades en un universo de oportunidades mínimas (Cortina, 2017; Castillo y Castro, 2011). Para lograr el objetivo, en un primer apartado recuperamos, a partir de varias fuentes internacionales y para fines panorámicos, indicadores socioeconómicos para los jóvenes en el mundo, incluimos esos indicadores como resultado y expresión de la violencia estructural en el sentido de Galtung (1981); en el segundo apartado rescatamos datos de una investigación realizada en instituciones de prevención social para señalar cómo una de las prácticas derivadas de la violencia estructural o injusticia, a saber, la reproducción de posturas o esquemas de pensamiento (estigmatizaciones, estereotipos, prejuicios, etcétera), pueden terminar responsabilizándolos y navegar en su contra, de manera particular en términos de sus derechos; en el tercer apartado reflexionamos acerca de las nociones de ciudadanía y sus posibilidades negadas para los jóvenes en riesgo en la entidad.

La lógica de este texto se inscribe en las temáticas de los estudios de la juventud y de las ciudadanías, particularmente en aquellas que conciben que, en 
contextos como los contemporáneos, en regiones como Latinoamérica (plagadas por la pobreza, la desigualdad y la inequidad), las ciudadanías de los jóvenes están condicionadas o enfrentan serias dificultades y riesgos (Niño, Valencia y Ruiz, 2017; Castillo y Castro, 2011).

\section{VIOLENCIA ESTRUCTURAL: CONTEXTO DE LOS JÓVENES}

Es importante destacar que, en estos principios del siglo XXI, muchos «jóvenes se autodefinen como una juventud sin futuro» (Torres, 2013: 2), con desesperanzas y con problemas sociales. Sin lugar a dudas es un lugar común definir el futuro como entidad para el disfrute juvenil en otro tiempo, pero que muchos jóvenes ni siquiera tengan en sus manos la posibilidad de soñar este lugar común es de resaltarse («cuando ni los lugares comunes se pueden ya soñar», podría ser el título de este callejón sin salida). Así, aunque el futuro no solo pertenezca a la juventud, puede leerse que este desencanto se debe al agotamiento del modelo tradicional de inserción social, el cual, si bien presentaba dificultades, les generaba certezas (Torres, 2013) ${ }^{4}$. Dichas certezas del futuro están agotadas, más bien es la incertidumbre la cualidad más sentida en el modelo liberal de acumulación capitalista de la actualidad, en que las élites gubernamentales de los Estados nacionales deben articularse con las élites económicas transnacionales para asegurar la acumulación, lo que Harvey (2012) llama acumulación por desposesión.

Por ejemplo, el informe Tendencias mundiales del empleo juvenil 2013 señala que «la tasa mundial de desempleo juvenil, que había disminuido del $12.7 \%$ en 2009 al $12.3 \%$ en 2011 , aumentó [...] al $12.4 \%$ en 2012 , y [...] $12.6 \%$ [...] en 2013. [...] [Asimismo indica que para] 2018 [...] [se esperaba que

\footnotetext{
${ }^{4}$ El modelo tradicional de inserción social se puede entender como aquel en que el Estado asume la responsabilidad de cubrir las necesidades de sus habitantes aun cuando ello presente problemas (de alcance, de recursos, de eficiencia y de eficacia), es decir, el Estado de bienestar.
} 
hubiera] una tasa mundial de desempleo juvenil de 12.8\%» (Sánchez-Castañeda, 2014: 136).

Para América Latina, estimaciones de la Organización Internacional del Trabajo (OIT) (Sánchez-Castañeda, 2014) dan cuenta que ha habido:

un incremento de la tasa de desempleo juvenil urbano de tres décimas entre 2012 y 2013 que oscila entre $14.2 \%$ y 14.5\%. Esto significa que había unos 6.6 millones de jóvenes desempleados en las áreas urbanas de la región en 2013. El desempleo juvenil supera en 2.25 veces la tasa de desempleo total y en 3.18 veces la tasa de desempleo de los adultos. [...] Además se estima que los jóvenes que no trabajan ni estudian son cerca de 21.8 millones, es decir, alrededor del 20\% de jóvenes en edad de trabajar. (Sánchez-Castañeda, 2014: 136-137)

Finalmente, el mismo autor apunta que, para el caso mexicano, datos de la Organización para la Cooperación y el Desarrollo Económicos (OCDE) muestran que, si bien la tasa de desempleo de 2012 fue menor a la de 2009, su comportamiento ha sido más o menos ascendente considerando como punto de arranque 2005. Estas cifras indican que el futuro, en empleo, no posee certezas.

En lo que se refiere a empleo precario o temporal (bajos salarios, nulos o limitados derechos laborales y explotación intensiva y extensiva de la mano de obra) (Hopenhayn, 2000; Corredor, 2004; Castillo, 2009; Ovejero, 2009; De la Garza, 2011; García y Guerrero, 2012), Sánchez-Castañeda indica que a nivel mundial, «el número de contratos temporales para los trabadores jóvenes casi se ha duplicado desde el inicio de la crisis económica [financiera internacional de 2008]» (2014: 135), lo que ha significado una disminución en su ya de por sí bajo nivel de vida $\mathrm{y}$, en muchos casos, su inserción en el trabajo precario de la economía informal (OIT-OMC, 2009; OIT, 2010). La calidad de los empleos y las formas vulnerables que adquieren los mismos generan descontento social y el deseo de migrar (OIT, 2017). 
Si bien la carestía en el empleo ilustra la incertidumbre del futuro, sucede lo mismo con las oportunidades de acceso a la educación. Por señalar algunos datos:

Según datos procedentes de 128 países, correspondientes al período 20102015, que abarcaban el 90\% de la población mundial en edad de cursar el segundo ciclo de la enseñanza secundaria, menos de uno de cada cuatro jóvenes había finalizado el segundo ciclo de secundaria en 40 países y menos de uno de cada dos en 60 países. Apenas 14 países presentaban una tasa de finalización de al menos el 90\%. (UNESCO, 2017: 151)

Hay hipótesis que pueden sustentar estos datos. Hay quienes argumentan que el acceso a la educación se ha dificultado en la medida de su privatización, lo cual llevaría a que miles de jóvenes se vean obligados a pagar o a quedar marginados si no tienen la suficiencia económica, lo que aumenta el riesgo de no poder insertarse en el mercado laboral o enfrentar condiciones de trabajo más precarias (Barbería, 2009).

Un vistazo para América Latina nos brinda un panorama más cercano a México como miembro de la OCDE. De acuerdo con Holm-Nielsen, Thorn, Brunner y Balán (2005), miembros del equipo de investigación del Banco Mundial:

La matrícula en educación superior se incrementó mucho en América Latina durante las últimas cuatro décadas. En el 2001 el 23\% de latinoamericanos entre 18-24 años estaba matriculado en instituciones postsecundaria. Esto quiere decir una tasa de crecimiento anual de la matrícula de 2.3\% desde 1985 [...] Pero a pesar del crecimiento impresionante, América Latina sigue rezagada de las economías más importantes. Entre los países de la OCDE el promedio de la tasa de matrícula en educación superior es actualmente de $56 \%$. (p. 40) 
Ciertamente, en varios países latinoamericanos, entre ellos México, haber asistido a la escuela no garantiza ni empleo ni mejores salarios para los jóvenes $\mathrm{y}$, en muchos casos, ni movilidad social, pero sí ofrece mayores elementos formativos para competir en mejores condiciones. No obstante, cuando el acceso a la educación es muy limitado, las posibilidades se vuelven restringidas y más cuando se es pobre, pues en esta circunstancia el acceso es casi nulo.

Finalmente, otra de las situaciones que viven los jóvenes del mundo, y que algunos autores lo ubican como parte de un ethos de la época global, es «el consumismo» (González, 2017: 28), el cual es invocado tanto por ricos como por pobres y alude tanto a cuestiones materiales como inmateriales. Si bien el consumismo no es propio de esta época, en la actual lleva a muchos jóvenes a problemas de tipo emocional debido a «la polarización de la riqueza y las brechas entre ricos y pobres» (González, 2017: 28) y sobre todo a su ubicación como parte de la valía de las personas (González, 2017), que hace que quienes no consumen algo enfrenten una «especie de declinación de [...] valores [pues sus] cuerpos [no] muestran en su exterior las marcas de la desigualdad hecha diferencia (Douglas e Isherwood, 1990), proyectando los estatus y las jerarquías, y las distinciones (Bourdieu, 1988)» (González, 2017: 28).

Ahora, aun cuando las condiciones anteriores parecen no tener responsables directos (con nombre y apellido) en tanto están presentes en la vida de muchos jóvenes, están relacionadas fuertemente con la injusticia social, la cual trae asociada una violencia estructural, «entendida como un tipo de violencia indirecta $[. .$.$] que en definitiva hacen que muchas de las necesidades humanas$ de la población no sean satisfechas» (Jiménez-Bautista, 2012: 33) y que, por cierto, como señala Galtung (1981), se vincula con otros dos tipos más de violencia: la violencia directa, entendida esta como la acción de implicar daño por parte de una persona a otra, y la violencia cultural, que hace referencia a aquellos elementos de la cultura, los cuales pueden justificar o legitimar alguna de las dos formas de violencia. 
Esta injusticia no es fruto únicamente de las dinámicas producidas por las relaciones de tipo económico, sino también de la opresión política que utiliza «mecanismos tan dispares como la discriminación institucional, [la] legislación excluyente de ciertos colectivos o la política fiscal y de gasto público regresiva» (La Parra y Tortosa, 2003: 62) que causan procesos de deprivación, por lo que tiene una relación con las decisiones gubernamentales.

Lo llamativo de esta injusticia radica en que es normalmente desconsiderada por las autoridades por omisión o por incompetencia y, al ocurrir ello, da espacio para el desarrollo de varias situaciones o prácticas que terminan afectando a los mismos jóvenes. Una de estas situaciones es la ampliación de las diferencias y desigualdades entre los jóvenes de los sectores pobres y ricos, pues, como señalan La Parra y Tortosa (2003):

Dentro de cada país existen grupos sociales que se benefician de una dinámica de incremento de la desigualdad con o sin apoyo del aparato institucional del Estado o del poder económico [...] [lo cual] se traduce en menores esperanzas medias de vida [para los que menos tienen] [...] [Y es que] las relaciones económicas, políticas, militares o culturales que se van configurando en la escala del sistema mundo y por tanto en la escala local sirven para estructurar el acceso a los recursos. (p. 69)

Otra de las situaciones a que da pie la desconsideración gubernamental de la injusticia social es la búsqueda de alternativas riesgosas entre los mismos jóvenes, tales como las migraciones de sus países pobres de origen a los países desarrollados, o de sus comunidades de origen a las ciudades, donde existen las posibilidades de ser objetos de violencia por parte de algunos grupos de la población, las policías y grupos del crimen organizado (Mendoza, 2011), «que incluyen una segregación, separación y marginación social progresiva» (Bauman, 2013: 9). No hay que olvidar, como lo señalan algunos autores, que los jóvenes que viven en contextos de violencia estructural también enfrentan el 
riesgo de ser sujetos de violencia cultural y directa a través de acciones diversas, como son los abusos de poder, el estigma, la discriminación, el racismo, la criminalización y la exclusión (García y Guerrero, 2012; Galtung, 1981). La violencia estructural también da pie a que los mismos jóvenes incursionen en actividades informales y/o fuera de la ley, pues en varias partes del mundo están viviendo no solo tiempos de crisis, sino condiciones de precariedad y aumentos desmedidos de la pobreza cuyos impactos viven al día (Gómez, 2013).

Asimismo, otra de las situaciones a que da pie la desconsideración gubernamental de la injusticia social es la reproducción de doctrinas o esquema de pensamiento conservadores, cuyos postulados visualizan a los jóvenes como responsables de sus acciones y todo lo que les sucede, aun cuando se considere que debajo de ellas subyacen factores estructurales. Es decir, da entrada a ideas de que los jóvenes son culpables directos de todo lo relacionado con ellos, como si estos fueran sujetos aislados o atomizados, sobre todo bajo la idea neoliberal de que ellos son poseedores del libre albedrío (Grima, 1999). Pero no, los jóvenes no eligen la violencia que viven o ejercen, estas son parte de las condiciones de pobreza y precariedad del contexto mundial y del ámbito local.

Las condiciones de pobreza y precariedad, generadas por la violencia estructural en que viven miles de jóvenes en México y como parte de este, en el ámbito local del Estado de México se pueden ilustrar con los siguientes datos del Consejo Nacional de Evaluación de la Política de Desarrollo Social (CONEVAL):

Entre 2010 y 2014 se registró un incremento en el número de jóvenes en condición de pobreza, al pasar de 46 a 47.1 por ciento. En términos absolutos esto significó un aumento de 16.8 a 17.5 millones de personas y si se compara con la población de entre 30 y 64 años — que ha mantenido su índice de pobreza alrededor de 40 por ciento en el mismo periodo - se evidencia una diferencia de alrededor de siete por ciento en desventaja para los jóvenes. (CONEVAL, 2016: 9) 
La propia Secretaría de Gobernación (2014) reconocía que: «Una de las principales barreras que enfrenta la población juvenil en el desarrollo de su vida es la pobreza, en México se estima que $45 \%$ de la población enfrenta algún tipo de pobreza, mientras que, de manera particular 44.9 del grupo de 12 a 29 años de edad enfrenta esta situación» (p. 26).

De acuerdo con cifras del CONEVAL, conforme a los últimos resultados presentados en el 2014, el Estado de México tenía un total de 8,2 millones de personas en condiciones de pobreza (Corona, 2017: s/p). Los más de 8 millones que viven en pobreza, contabilizados por el CONEVAL, representaron casi al 50 por ciento de la población en el Estado de México, y en los últimos años la cifra ha ido en aumento. En 2010, los pobres fueron equivalentes a 42.9 por ciento del todos los mexiquenses; para 2012 incrementaron a 45.3 por ciento, y en 2014 a 49.6 [...] El Estado de México obtuvo también, de 2012 a 2014, el segundo lugar entre los cinco estados que más acrecentaron el número de habitantes en pobreza extrema (Tirado, 2016: s/p).

\section{JóVENES EN RIESGO, JÓVENES VULNERADOS. ALGUNOS EFECTOS SOBRE DOCTRINAS CONSERVADORAS EN TORNO A ELLOS}

En este apartado recuperaremos algunos discursos captados dentro de una investigación llevada a cabo en 2016 en tres instituciones de prevención social del Estado de México (denominadas Preceptorías Juveniles Regionales de Reintegración Social), la cual tuvo por objeto hacer una interpretación de ciertas ideas y/o posturas manejadas por dichas autoridades sobre la prevención de conductas antisociales entre los jóvenes considerados en riesgo y la reintegración de otros más que habían cumplido ciertos años de internamiento por la comisión de algunas de ellas. En dicha investigación se realizaron tres entrevistas a autoridades — una de cada preceptoría—, además de otra persona que había trabajado en una de ellas, y observaciones de campo de dos torneos (uno de fútbol y otro de ajedrez), los cuales fueron organizados por las propias preceptorías y 
autoridades del gobierno estatal como parte de una semana llamada de la prevención ${ }^{5}$.

La idea de retomar parte de los discursos vertidos por las autoridades investigadas radica, a diferencia de otros trabajos que hemos desarrollado, en mostrar parte de los efectos que pueden tener la reproducción de doctrinas o esquema de pensamiento en el abordaje de ciertos grupos juveniles y cómo estos, al final, pueden navegar en su contra, particularmente en términos de la representación de sus personas.

Dentro del conjunto de discursos captados en la investigación mencionada, una de las cuestiones que más llamó nuestra atención fue la relacionada con el tipo de jóvenes que abordan. Si bien las autoridades de las instituciones preventivas consideran que todos los jóvenes de la entidad son objeto de su atención, en tanto cada uno de ellos está en riesgo de adoptar o manejar conductas antisociales, conciben que su foco de interés es un gran número de jóvenes de los sectores populares, quienes, desde su lectura, estarían en un riesgo mayor. Ello debido a las condiciones familiares y materiales adversas que normalmente les afecta y que los pone en esa condición.

La respuesta a la pregunta ¿qué tipo de jóvenes llegan, qué características socioeconómicas, conductuales tienen? ilustra respecto a las consideraciones previas:

Normalmente de bajos recursos, socialmente contaminados, de familias disfuncionales, mal integradas, generalmente ese es el perfil: no [llegan de familias ricas] [...] no porque vivimos en un país de los más corruptos a nivel mundial $[\ldots]$ así que ellos ni siquiera son procesados [...]. (Entrevista 06/04)

\footnotetext{
${ }^{5}$ Las personas entrevistadas tenían los siguientes cargos administrativos: uno era presidente de una preceptoría; otro, director de área de otra de ellas y otro más encargado de otra área de la tercera preceptoría, lo cual les permitía conocer varios de los asuntos que se tratan en las preceptorías. El cuarto entrevistado trabajó también en un puesto similar. Los fragmentos de las entrevistas que se refieren en el artículo se consignan con el día y el mes (abril) en que se realizaron, aunque se guarda la identidad de los entrevistados para mantener su anonimato.
} 
La respuesta y argumentación previas traen asociada una valoración «crítica» de las familias de los sectores populares, en tanto las ubica como problemas o al menos con mayores problemas que las de otros sectores, la cual las deja en una condición negativa: la de poseer mayor susceptibilidad de «contaminarse». Dicha condición no solo afecta a las familias de dichos sectores, a quienes se les llega incluso a estigmatizar, sino a los jóvenes de esos mismos sectores en tanto se les ubica como con problemas por ser parte de esas familias. El nacer en una familia pobre agrega el problema de la susceptibilidad a generar jóvenes conflictivos, con problemas sociales asociados a la pobreza, tales como la violencia o la drogadicción.

Otra de las cuestiones que captó nuestra atención, vinculada con lo anterior, fue la relacionada con las denominadas conductas patógenas familiares. De acuerdo con las autoridades, estas conductas - entre las que se incluyen abandonos paternales, vicios, amistades, divorcios - son unas de las principales causas que ponen a los jóvenes de los sectores populares en las rutas de las conductas antisociales, debido a que les afectan sus personalidades.

$[\ldots]$ hay problemas familiares en general y eso los conlleva a cometer, pues conductas que en su momento podrían ser patógenas y concluyen en materia de salud pública ¿sí? Entonces lo que hacen las Preceptorías es identificar, con ayuda de los directivos, a estos jóvenes, obviamente a concientizar a los padres de familia a efecto de que acudan a recibir un tratamiento, una orientación, un tratamiento terapéutico [...]. (Entrevista 05/04)

Más allá de que estas conductas tengan o no el influjo que señalan las autoridades en la antisocialidad de los jóvenes, lo que llama la atención es que con ello llevan a este último fenómeno a un nivel esencialmente psicológico, donde se exaltan los aspectos familiares como factores desencadenantes de conductas antisociales o, para decirlo de otra manera, donde se cree que hay una 
relación estrecha entre lo que ocurre en las familias y las conductas de los jóvenes. Lo cual no es un asunto menor, pues con esto se olvida que la precariedad, la pobreza y vulnerabilidad son también factores que contribuyen a la generación de contextos de violencia en los que se involucran las juventudes, al tiempo de que se les responsabiliza a las familias a quienes se les sojuzga de sus conductas «patológicas». El punto nodal es que este tipo de prejuicios o supuestos sociales llevan a pensar que las familias pobres producen el tipo de conductas antisociales vinculadas a las drogas, el mercado negro y la violencia juvenil, quedando la pregunta, no indagada por estas instituciones, sobre qué tipo de conductas que producen algunas familias ricas (jóvenes indiferentes, ejecutores de abuso del poder, corrupción, etcétera).

En lo que refiere a las funciones de las instituciones preventivas, se señalan dos acciones: una de prevención y otra de readaptación (cuando los jóvenes ya han delinquido). En la explicitación de dichas funciones aparecen dos elementos fundamentales: la sospecha y una figura de autoridad. La sospecha se presenta cuando se menciona que miembros de la institución acuden a las escuelas, de los sectores populares, para identificar a jóvenes en riesgo, así como cuando dicen que se encargan de vigilar el proceso de readaptación de los jóvenes que delinquieron en algún momento. Por otro lado, la figura de autoridad aparece cuando definen concretamente quiénes son sujetos en riesgo y qué tareas deben hacer los jóvenes que están en proceso de readaptación.

En forma preventiva, nosotros lo que hacemos es ir a las escuelas como tal para localizar el tema principal del medio que lo rodea en la escuela y chavos que hayan cometido algún delito o sospechosos, ahí [...] Entonces ahí podemos identificar a los adolescentes en estado de riesgo de que cometan alguna conducta como un sospechoso o que lo vieron con drogas, lo vieron fumando mariguana o que lo acusan de robar un celular; normalmente nos llegan aquí por robo, es el delito que más se comete y el que nosotros vemos más. (Entrevista 06/04) 
Otro tipo de joven que tenemos es el joven que ya desafortunadamente ya pisó el MP (se refiere al Ministerio Público), ya lleva un procedimiento en el juzgado y el juez ha dictado un tipo de resolución y de aquí pasan a una sentencia ¿por qué? Porque aquí ya delinquió, ya cometió una conducta social, que ya se tipificó como delito y, bueno, ahí ya a criterio del juez el tiempo que el joven debe de venir ya a recibir una terapia [...] él ya tiene que venir a fuerza con sus papás, obviamente nosotros ya cumplimos con la instrucción de los $[\ldots]$ que nos envía el juez: «sabes que vas a estar aquí seis meses, un año o año y medio» $[\ldots]$ Nosotros tenemos que acatar la instrucción que nos envía el juzgado, obviamente nosotros enviamos al juzgado de manera mensual, el avance y evolución del joven, ¿sí? Y hasta en tanto, si es favorable este avance, esta evolución, el juez determina si el joven ya puede darse de alta al joven de su tratamiento. (Entrevista 05/04)

Desde estas posturas los derechos de los jóvenes parecen quedar vulnerados, pues en el caso de la sospecha no se les considera más que en negativo, por lo que pueden hacer, pero en forma perjudicial. Tanto es así que, en el caso de los jóvenes en proceso de reintegración, se les sigue vigilando; mientras que en el caso de la figura de autoridad, su beneficio queda al albedrío de las autoridades, quienes definen o señalan cuándo están en riesgo y cuándo ya están totalmente reintegrados a la sociedad, lo cual, además de no cuestionar el funcionamiento de la sociedad en su conjunto, lleva a pensar que la conducta antisocial es más el producto de una decisión individual que una reacción sistémica. El enfoque reduccionista es evidente y la falta de institución para una sociedad que clama ayuda, también lo es.

Las autoridades asimismo asumen una postura «funcional» respecto a la estructura organizacional de las instituciones preventivas. De acuerdo con ellas, si bien dicha estructura llega a presentar problemas por los recursos con que disponen estas instituciones, es suficiente para abordar a los jóvenes de una manera integral. Ello en razón de que consideran su aspecto emocional, su 
condición familiar, su educación y su salud, además de su condición legal cuando se requiere.

Cada Preceptoría está integrada por un presidente, su servidor (sic), un secretario de acuerdos, un psicólogo, un trabajador social, un pedagogo, un médico, y un promotor social; obviamente el joven tiene que pasar por cada una de las áreas y cada una emite su diagnóstico de manera mensual para ver su avance y su evolución. (Entrevista 05/04)

Lo que da cuenta esta postura es que, desde la mirada de las autoridades, los jóvenes son sujetos atomizados, casi aislados, que requieren una atención centrada en sus personas o en sus familias. Desde un ángulo institucional es funcional, pues con ello, al tiempo de desconsiderar las fallas del Estado para abordarlos (y la actual economía de mercado que pretende por todos los medios publicitarios el consumismo engarzado a relatos de privilegio y status alcanzados en medio de la abundante pobreza), les ofrece cosas muy particulares que no rebasan niveles personales o familiares.

Finalmente, resulta importante mencionar la convicción que parecen tener las autoridades sobre las acciones que llevan a cabo con los jóvenes de los sectores populares y que llevan a señalar cosas como las planteadas por una directora de una preceptoría, participante en uno de los torneos de la semana de la prevención, después de la conclusión de uno de ellos:

El deporte tiene que alejarnos de muchos vicios. Lo importante es involucrarnos en actividades productivas. No dejen el deporte, ustedes pueden ser algunos de los seleccionados, es un sueño, luchen por sus sueños. (Nota de campo 16/04)

En términos generales, las autoridades consideran que las acciones llevadas a cabo ayudan a que los jóvenes no se orienten a las conductas antisociales, si es 
que no han cometido alguna, o se restrinjan de hacerlas, si ya cometieron otras, debido a que apuntan a hacerlos conscientes, a enseñarles a manejar su tiempo y a volverlos más sociales, entre otras cosas. Pero también debido a que consideran a sus familias en varias acciones e incluso a sus entornos inmediatos, para hacerlos responsables de la formación de los jóvenes. Asimismo, señalan que el éxito de dichas acciones se visualiza de manera cualitativa cuando observan los cambios en las conductas de los jóvenes y sus familias, así como en el número de jóvenes que llegan a las instalaciones de la Preceptorías.

Independientemente de que ocurra lo que se considera en el párrafo previo, esta postura deja entrever nuevamente un supuesto institucional individualista, según el cual, para atender las conductas antisociales se debe trabajar desde un nivel psicológico que considere los círculos concéntricos de los jóvenes. Ello, sin embargo, y como ya sugerimos antes, manifiesta una postura reduccionista, puesto que apunta a hacer entender a los jóvenes y sus familias que los problemas están en sus individualidades y no en las condiciones estructurales, lo que significa que no buscan cambios profundos que les beneficien a todos ellos en tanto grupo etario, sino solamente a unos en tanto entes aislados que, al final de cuentas, termina por destacar logros individuales y no un bienestar general.

\section{JÓVENES EN RIESGO, JÓVENES VULNERABLES:}

\section{CIUDADANÍA NEGADA}

A partir de lo dicho, surgen dos preguntas: por un lado, ¿los jóvenes y, de manera particular, los jóvenes de los sectores menos favorecidos tienen la posibilidad de constituirse como ciudadanos?, y por otro, ¿es posible que los jóvenes de los sectores populares en México, considerados en riesgo de cometer conductas antisociales, puedan apuntar a una ciudadanía cuando enfrentan condiciones adversas y concepciones que los ubican como problemáticos o en negativo? Para contestar estas preguntas, en esta sección explicaremos primeramente a qué alude el término ciudadanía para, posteriormente, en la 
última sección del artículo, esgrimir algunas reflexiones en torno a ello, aunque advertimos desde este momento una postura escéptica.

La noción de ciudadanía alude a la titularidad de derechos, pero también de obligaciones que tienen los individuos en tanto miembros de una comunidad (Benedicto y Morán, 2002), lo cual supone una integración de elementos que asegura «que cada cual sea tratado como un miembro pleno de una sociedad de iguales» (Kymlicka y Norman, 2002: 4).

Para algunos especialistas, seguidores de la propuesta original de T.S. Marshall, la ciudadanía empieza con el reconocimiento creciente de los derechos de los individuos, a saber, civiles, políticos y sociales, «que determina el sentido de pertenencia a la comunidad nacional y favorece la participación en la vida social» (Bojórquez, 2005: 79); para otros especialistas, en cambio, inicia con la responsabilidad que deben asumir los propios individuos con la sociedad para su contribución a ella; y para otros más, con la conjunción de ambos elementos (derechos y obligaciones). No obstante, la mayoría de ellos coinciden en que la noción en sí reconoce lo planteado por Touraine, a saber, «la capacidad del individuo de sentirse responsable del buen funcionamiento de las instituciones, en simetría con la obligación de las instituciones de respetar los derechos de los hombres y mujeres» (Touraine, citado en Bojórquez, 2005: 80). Ello debido a que considera tanto un ámbito objetivo como otro subjetivo, tal como lo expresa Bojórquez:

El primero se relaciona con las condiciones externas que garanticen un cumplimiento efectivo del derecho que le asiste a la persona. Estas condiciones son de orden legal, institucional y financiero. El orden legal posibilita la exigibilidad del derecho, el institucional crea los organismos necesarios para su ejercicio y el orden financiero permite el ejercicio del derecho. El ámbito subjetivo [por otro lado] se refiere a la habilitación de la persona para ejercer sus derechos. Esto implica la necesidad de conocerlos para participar en la creación de reglas de conducta y el desarrollo de capacidades personales acordes con el ejercicio y la protección de sus derechos. (Bojórquez, 2005: 80) 
Ahora, aunque la ciudadanía remite originalmente a la pertenencia a una nación, «el impacto de la posmodernidad en el campo cultural, de la globalización en el campo político, y de la revolución de la información en el campo tecnológico [de los últimos años]» (Hopenhayn, citado en Bojórquez, 2005: 85) ha llevado a plantear su «ensanchamiento» para considerar una nueva serie de derechos (como los culturales y los de diferencia y promoción de la diversidad) y otras obligaciones de los individuos, pero también su «achicamiento» para tomar en cuenta otros derechos y obligaciones locales, lo cual, en el fondo, no cambia su esencia, puesto que apuntan al bienestar de los individuos y al vínculo que existe entre ciudadanía y desarrollo.

Finalmente, vale indicar que para hablar de una plena ciudadanía se requiere ir más allá de los derechos civiles y políticos, y orientarse en una ruta de democratización social o ¿democracia social? ${ }^{6}$, pues solo a partir de ello se puede tener garantizada la libertad de vivir por encima de la barrera de la necesidad (Sen, 2000), lo cual permite, a su vez, ejercer los derechos civiles, los derechos políticos y los derechos culturales bajo una lógica de interacción definida por mínimos civiles que compartimos todos (Cortina, 2017). Esta condición parece un poco utópica, en tanto la constante en varios países parece mostrar lo contrario, empero, es importante trabajarla en la medida que constituye la plataforma para una sociedad ciudadanizada en la que todos podemos embarcarnos.

\section{DISCUSIÓN Y CONCLUSIONES}

\footnotetext{
${ }^{6}$ La teoría de la Democracia Social «está marcada por: Derechos fundamentales en el ámbito social y económico; constitución de la sociedad conforme a los derechos fundamentales (tanto en el plano formal como en el real); y vigencia formal y aplicación real de derechos de libertad negativa y positiva» (Gombert, et al., 2010: 97).
} 
Si retomamos las preguntas planteadas en el apartado anterior, podemos decir que los jóvenes de los sectores populares en México y el Estado de México parecen tener menos posibilidades de constituirse como ciudadanos que los de otros sectores, en razón de las siguientes cuestiones.

En primer lugar, en razón de que los gobiernos en turno enfatizan particularmente - aunque con sus fallas - en los derechos civiles y políticos, los cuales, si bien son importantes, no son los únicos para lograr una plena ciudadanía, pues también son necesarios los derechos sociales y culturales, tal como lo alude Bobbio (1992) cuando señala que «una cosa es la democratización del Estado [...] y otra cosa es la democratización de la sociedad» (p. 43). Y es que en México y el Estado de México, cerca del cincuenta por ciento de la población total vive en condiciones de precariedad, pobreza y vulnerabilidad que hacen difícil el ejercicio o cumplimiento de la ciudadanía social como lo alude implícitamente el Pacto Internacional de Derechos Económicos Sociales y Culturales; y por ello tampoco cuentan con el capital cultural para ejercer realmente sus derechos civiles y políticos. Ahora, del total de la población que vive en esas condiciones, millones de ellos son jóvenes de los sectores populares que no tienen la posibilidad de acceder a la educación ni a un trabajo con derecho a seguridad social y servicios de salud, tampoco a una vivienda digna ni a un barrio o colonia que garantice el acceso a la oferta cultural en su totalidad.

En segundo lugar, porque en México y el Estado de México, aunque en sus discursos las autoridades de gobierno digan que existen políticas y programas de generación de empleo y de desarrollo social para todos, los datos oficiales evidencian cierta incongruencia de esos discursos con la realidad social en que viven o sobreviven millones de jóvenes. Por ejemplo, mientras los autores de este artículo revisamos las sugerencias de los dictaminadores anónimos, identificamos una nota periodística (Martínez, 8 de abril de 2019), en la que se indicaban algunas complicaciones que tenía el diseño de la plataforma de «Jóvenes construyendo el futuro», un programa social nacional para jóvenes del gobierno encabezado por Andrés Manuel López Obrador, pero sobre todo la idea 
de que no había sido pensada para jóvenes de los sectores con menos recursos, quienes están excluidos por no tener acceso a la tecnología. De hecho, se indicaba que una de las autoridades federales lo había admitido, manifestando lo siguiente: «Vamos a trabajar con los jóvenes de las zonas urbanas marginadas, a fin de que el programa no los excluya de nuevo» (Martínez, 8 de abril de 2019). Y es que, si bien han existido y existen programas para los jóvenes, muchos de ellos no llegan a todos los sectores, pues son de carácter asistencialista, focalizados (en ciertos sectores) y por lo tanto paliativos, de uso político.

En tercer lugar, para el caso del Estado de México y según creemos para otras partes del país, en virtud de que las autoridades y funcionarios de gobierno despliegan una serie de discursos a través de los cuales individualizan y culpabilizan a los mismos jóvenes respecto a la precariedad y pobreza en que viven, argumentando a la vez que son resultado del fracaso familiar y personal, imponiéndoles, a fuerza de la repetición incansable, la interiorización de esos discursos.

Lo anterior puede entenderse como resultado, en parte, de las visiones adultocéntricas, las cuales son coincidentes con las posturas gubernamentales y que llevan a que se construyan imaginarios de las y los jóvenes, y sus familias, muchos de ellos desfavorables. Ta como lo observamos en los discursos sobre las Preceptorías juveniles analizados previamente, que señalan a las familias disfuncionales como responsables de su disfuncionalidad y como la causa de las condiciones de precariedad, pobreza y vulnerabilidad de miles de jóvenes con «conductas antisociales» y en riesgo de la comisión de algún delito; aludiendo a trayectorias biografiadas, individualizadas en contextos de inseguridad y falta de certezas. En ese sentido, lo relevante de estos imaginarios es que con ellos las autoridades evaden su responsabilidad de generar condiciones de desarrollo social y asumen solo el papel de vigilantes y gendarmes del «orden» (un orden no cuestionado), poniendo bajo sospecha o todo joven que sea parte de esas condiciones sociales de vida. En otras palabras, en lugar de políticas de desarrollo 
social, se implementan políticas de criminalización y medicalización para miles de jóvenes desde la perspectiva psicopatológica individualista.

En cuarto lugar, en razón de que las autoridades electorales como las del Estado de México ponen más énfasis en convocar a los jóvenes (que están por cumplir 18 años o que los acaban de cumplir), a través de la televisión y la radio de señal abierta, comercial e institucional, regularmente y de manera enfática cuando se acerca algún proceso electoral, a que acudan a las oficinas más cercanas del Instituto Nacional Electoral (INE) a tramitar su credencial de elector para convertirse formalmente en ciudadanos y elegir a los candidatos que serán sus representantes, que en otros aspectos. También son frecuentes los promocionales de la Legislatura en turno, así como del Senado de la República y las labores de sus integrantes: diputados y senadores «en pro» de la población y los ciudadanos. Sin embargo, la ciudadanía civil y política no se adquiere solo con la credencial para votar, sino con la cultura política que otorga el capital cultural en condiciones sociales que dan acceso a la educación media superior y superior.

Finalmente, en razón de existir diversas formas de violencia estructural, violencia simbólica y violencia directa; y también corrupción e impunidad como parte de esas violencias, las cuales crean un clima para que, por ejemplo, en el Estado de México miles de jóvenes menores de 18 años no logren ejercer su ciudadanía social al carecer de oportunidades de desarrollo de su potencial individual, y en su lugar sí encuentren muchos riesgos, entre ellos, los que los estigmatizan desde las instituciones, que los potencian como menores infractores, y los que ya han cometido algún delito se enfrenten al encierro para la «readaptación y reintegración familiar y social». Un encierro que, dependiendo del delito y de los logros de las Preceptorías y de las familias de los jóvenes implicados, los puede convertir en delincuentes que pasan del encierro (en la Escuela de readaptación), al encierro en algún Centro de Readaptación Social, Estatal o Federal (CERESO o CEFERESO), para adultos. 
No hay que olvidar que, muchas veces, la prevención del delito en jóvenes en riesgo y la coadyuvancia en la readaptación familiar y social para el caso de quienes cometieron algún delito no se logran a través de las instituciones preventivas porque, aunque en el discurso de las autoridades entre sus funciones contribuyen a construir tejido social, la complejidad de la violencia estructural e institucional les rebasa y les coloca como uno más de sus eslabones, al tener en la mira a los jóvenes en riesgo como potenciales delincuentes, medicalizando su cotidianidad. Lo único que puede construir tejido social, ciudadanía política y ciudadanía social es la política de desarrollo social que genere realmente oportunidades de educación media superior y superior, empleo y condiciones de vida dignas para millones de jóvenes en el Estado de México.

RECIBIDO: 6 DE AGOSTO DE 2018

ACEPTADO: 5 DE ABRIL DE 2019

\section{BibLIOGRAFÍA}

ABAD, M. (2002). Posibilidades y limitaciones de la participación juvenil para el impacto en la agenda pública. El caso del Consejo Municipal de Juventud en Medellín. Última Década, 17, 65-94.

BARBeríA, J. L. (2009). «Generación "ni-ni”: ni estudia ni trabaja». El País, España (versión digital), 22 de junio. Disponible en: http://www.elpais.com/articulo/sociedad/Generacion/nini/estudia/trabaja/elpepu soc/20090622elpepisoc_1/Tes

Bauman, Z. (2013). La globalización. Consecuencias humanas. México, DF: Fondo de Cultura Económica.

Benedicto, J, y MorÁn, M. L. (2002). La construcción de una ciudadanía activa entre los jóvenes. Madrid: Instituto de la Juventud. Disponible en: http://www.injuve.es/observatorio/valores-actitudes-y-participacion/laconstruccion-de-una-ciudadania-activa-entre-los-jovenes 
BobBio, N. (1996). El futuro de la democracia. México, DF: Fondo de Cultura Económica.

Bojórquez, N. (2005). Ciudadanía. En Y. Corona y N. Del Río (Coords.), Antología del Diplomado. Derechos la Infancia. Infancia en riesgo, Tomo I. México, DF: Universidad Autónoma Metropolitana.

CAstillo, D. (2009). Los nuevos trabajadores precarios. México, DF: Universidad Autónoma del Estado de México/M.A. Porrúa.

CAstillo, A. y CASTRO, X. (2011). El rostro de la violencia social y estructural: la delincuencia y la pobreza como expresiones distintas de una vulnerabilidad común. Rev. Ciencias Sociales, III-IV, 113-124. Disponible en: https://revistas.ucr.ac.cr/index.php/sociales/article/viewFile/3863/3736

CONEVAL. (2016). Informe de Evaluación de la Política de Desarrollo Social. Principales hallazgos. Disponible en: http://www.Principales-Hallazgos-IEDPS2016.pdf

Corona, S. (2017). «El reto, en EdoMex, de enfrentar la pobreza». El Economista, 20 de abril. Disponible en: https://www.eleconomista.com.mx/politica/El-reto-en-Edomex-de-enfrentar-lapobreza-20170420-0032.html

Corredor, C. (2004). El papel del trabajo en el contexto de la globalización. Revista PORIK AN, 9. Disponible en: http://www.maestriadesarrollo.com/porikan/porik-9

Cortina, A. (2017). Aporofobia, el rechazo al pobre. Un desafio para la democracia. Barcelona: Paidós.

De Hoyo, R., Halsey, R. y SzÉKely, M. (2016). NINIS en América Latina. 20 millones de jóvenes en busca de oportunidades. Washington DC: Grupo Banco Mundial. Disponible en: https://openknowledge.worldbank.org/handle/10986/22349

De la GarZa, E. (2011). Hacia un concepto ampliado de trabajo. Del concepto clásico al no clásico. Madrid: Siglo XXI. 
GaltunG, J. (1981). Contribución específica de la irenología al estudio de la violencia y su tipología. En La violencia y sus causas (pp. 91-106). París: UNESCO.

Disponible

en:

www.unesdoc.unesco.org/images/0004/000430/043086so.pdf

GiMÉNEZ, M. DEL C. (2009). Reseña de: Trabajo, subjetividad y ciudadanía: paradojas del empleo en una sociedad en transformación. De Eduardo Crespo, Carlos Prieto y Amparo Serrano (Eds.). Anuario de Psicología, 40, 2, 289-298. Disponible en: http://revistes.ub.edu/index.php/Anuariopsicologia/issue/view/735/showToc

Gombert, T., Bläsius, J., DAhm, J., Krell, C., Timpe, M. et al. (2010). Manual de la Democracia Social 1. Fundamentos de la democracia social. Buenos Aires: Fundación Foro Nueva Sociedad. Disponible en: www.library.fes.de/pdf-files/iez/07419.pdf

GonZÁlEZ, F. (2017). Ciudadanías en tiempos violentos; la declinación de las reglas sociales y la emergencia de nuevos procesos cognitivos. En J. J NiÑO, P. A. VAlencia y G. RuIZ (Coords.), Ciudadanías emergentes y transiciones en América Latina. Medellín: Universidad de Medellín/Universidad Autónoma del Estado de México.

GRIMA, J. M. (1999). Una mirada sociológica. En J. M. GRIMA y A. LEFUR, ¿Chicos de la calle o trabajo chico? Buenos Aires: Lumen Humanitas.

HARVEY, D. (2012). La condición de la posmodernidad. Investigación sobre los orígenes del cambio cultural. Buenos Aires: Amorrortu.

Holm-Nielsen, L. B., Thorn, K., Brunner, J. J. y BALÁn, J. (2005). Desafíos regionales e internacionales para la educación superior en América Latina. En H. De Wit, I. C. JARAMillo, J. GACEL-Ávila y J. KNIGHT, Educación superior en América Latina. La dimensión internacional (pp. 39-70). Bogotá: Banco Mundial/Mayol Ediciones S.A. Disponible en: http://documents.worldbank.org/curated/en/797661468048528725/pdf/343530S PANISH0101OFFICIAL0USE0ONLY1.pdf 
HopenHAYN, M. (2000). Nueva secularización, nueva subjetividad: el descentramiento del trabajo y de la política. Revista Estudios Sociales, enero, 005 .

Disponible

en:

https://issuu.com/publicacionesfaciso/docs/revista_estudios_sociales_n_5 JiMÉNEZ-BAUTISTA, F. (2012). Conocer para comprender la violencia: origen, causas y realidad. Convergencia. Revista de Ciencias Sociales, 19(58), 13-52. Disponible en: https://convergencia.uaemex.mx/issue/view/110

KymlickA, W. y Norman, W. (1997). El retorno del ciudadano. Una revisión de la producción reciente en teoría de la ciudadanía. La Política. Revista de estudios sobre el estado y la sociedad, 3, 5-40. Disponible en: https://dialnet.unirioja.es/revista/850/A/1997

LA PARra, D. y Tortosa, J. M. (2003). Violencia estructural, una ilustración del concepto. Documentación Social, 131, GEPYD Grupo de Estudios de Paz y Desarrollo, Universidad de Alicante. Disponible en: www.ugr.es/ fentrena/Violen.pdf

MARTíneZ, F. (2019). «Muro burocrático en el programa de empleo a jóvenes». La Jornada, 8 de abril. Disponible en: https://www.jornada.com.mx/ultimas/2019/04/08/muro-burocratico-en-elprograma-de-empleo-a-jovenes-5364.html Mendoza EnRíqueZ, H. (2011). Los estudios sobre la juventud en México. Espiral 18(52), 193-224. Disponible en: http://www.espiral.cucsh.udg.mx/index.php/EEES/issue/view/91/showToc Niño, J. J., VAlencia, P. A. y Ruiz, G. (2017). Ciudadanías emergentes y transiciones en América Latina ( $1^{\mathrm{a}}$ ed.). Medellín: Universidad de Medellín. Disponible en: https://www.latinamericanbooks.com/pages/books/62914/josejavier-nino-martinez-coords-paula-andrea-valencia-londono-gabriel-ruizromero/ciudadanias-emergentes-y-transiciones-en-america-latina OCHOA LEÓN, S. M. (2008). Construcción de ciudadanía y derechos sociales. Documento de trabajo No. 38. Cámara de Diputados LXIII Legislatura, México, Centro de Estudios Sociales y de Opinión Pública. Disponible en: 
http://www3.diputados.gob.mx/camara/content/.../file/Construccion_ciudadania _d38.pdf

OIT-OMC. (2009). La globalización y el empleo en el sector informal en los países en desarrollo. Disponible https://www.wto.org/spanish/res_s/booksp_s/jobs_devel_countries_s.pdf OIT. (2010). Tendencias Mundiales de Empleo Juvenil 2010. Edición especial sobre las repercusiones de la crisis económica en los jóvenes. Ginebra: Oficina Internacional del Trabajo. en: http://www.ilo.org/global/research/global-reports/global-employmenttrends/WCMS_598679/lang--es/index.htm . (2017). Perspectivas sociales y del empleo en el mundo. Tendencias 2017. Disponible en: http:/www.ilo.org/global/research/globalreports/weso/2017/WCMS_540901/lang--es/index.htm SÁNCHEZ-CASTAÑEDA, A. (2014). Los jóvenes frente al empleo y desempleo: la necesaria construcción de soluciones multidimensionales y multifactoriales. Revista Latinoamericana de Derecho social, 19, julio-diciembre, 133-162. Disponible en: https://revistas.juridicas.unam.mx/index.php/derechosocial/issue/view/474

SEN, A. (2000). Desarrollo y libertad. Barcelona: Planeta.

TiRADO, J. (2016). «Edomex aumenta en $28 \%$ la pobreza extrema». El Financiero, 29 de enero. Disponible en: http://www.elfinanciero.com.mx/nacional/edomex-aumenta-en-28-la-pobrezaextrema.html

TORRES MAESTRO, I. (2013). Juventud(es) y violencia estructural. En VII Jornadas de Jóvenes Investigadores. Instituto de Investigaciones Gino Germani, Facultad de Ciencias Sociales, Universidad de Buenos Aires, Argentina. Disponible en: https://www.aacademica.org/000-076/26

UNESCO. (2017). Informe de seguimiento de educación en el mundo 2017/8. Rendir cuentas en el ámbito de la educación: cumplir nuestros compromisos. París: Ediciones UNESCO. Recuperado de: www.unesco.org 


\section{SITIOS WEB}

Directorio De Preceptorías JuVEniles de ReINTEgración Social. Disponible en: http://dgprs.edomex.gob.mx/directorio_preceptorias EDOMEX INFORMA. (2013). «Fomentan en preceptorías juveniles oficios y bellas artes para combatir conductas antisociales», 21 de noviembre. Disponible en: http://edomexinforma.com/2013/11/fomentan-en-preceptoriasjuveniles/

Secretaría de Gobernación de MéXico. (2014a). Programa Nacional de la Juventud 2014-2018. «Introducción» y «Visión general». Diario Oficial de la Federación, 30 de abril. Disponible en: http://www.dof.gob.mx/nota_detalle.php?codigo=5343095\& fecha $=30 / 04 / 2014$ . (2014b), Programa Nacional de la Juventud 2014-2018. «Indicadores».

Diario Oficial de la Federación, 30 de abril. Disponible en: http://www.dof.gob.mx/nota_detalle.php?codigo $=5343095 \&$ fecha $=30 / 04 / 2014$ 